ANNALES

POLONICI MATHEMATICI

LXXVII.1 (2001)

\title{
Bifurcation in the solution set of the von Kármán equations of an elastic disk lying on an elastic foundation
}

\author{
by JoAnNA JANCZEWSKA (Gdańsk)
}

\begin{abstract}
We investigate bifurcation in the solution set of the von Kármán equations on a disk $\Omega \subset \mathbb{R}^{2}$ with two positive parameters $\alpha$ and $\beta$. The equations describe the behaviour of an elastic thin round plate lying on an elastic base under the action of a compressing force. The method of analysis is based on reducing the problem to an operator equation in real Banach spaces with a nonlinear Fredholm map $F$ of index zero (to be defined later) that depends on the parameters $\alpha$ and $\beta$. Applying the implicit function theorem we obtain the following necessary condition for bifurcation: if $(0, p)$ is a bifurcation point then $\operatorname{dim} \operatorname{Ker} F_{x}^{\prime}(0, p)>0$. Next, we give a full description of the kernel of the Fréchet derivative of $F$. We study in detail the situation when the dimension of the kernel is one. We prove that $(0, p)$ is a bifurcation point by the use of the Lyapunov-Schmidt finite-dimensional reduction and the Crandall-Rabinowitz theorem. For a one-dimensional bifurcation point, analysing the Lyapunov-Schmidt branching equation we determine the number of families of solutions, their directions and asymptotic behaviour (shapes).
\end{abstract}

1. Introduction. Let $\Omega=\left\{(u, v) \in \mathbb{R}^{2}: u^{2}+v^{2}<1\right\}$. We will denote by $C_{0,0}^{4, \mu}(\bar{\Omega})$, for each $\mu \in(0,1)$, the subspace of functions $f$ from the real Hölder space $C^{4, \mu}(\bar{\Omega})$ that satisfy the boundary conditions

$$
\left.f\right|_{\partial \Omega}=\left.\Delta f\right|_{\partial \Omega}=0
$$

where $\Delta$ is the Laplace operator. For all $C^{2}$ functions $f, g: \bar{\Omega} \rightarrow \mathbb{R}$ and every $C^{4}$ function $h: \bar{\Omega} \rightarrow \mathbb{R}$ we define

$$
\begin{aligned}
{[f, g] } & =\frac{\partial^{2} f}{\partial u^{2}} \frac{\partial^{2} g}{\partial v^{2}}-2 \frac{\partial^{2} f}{\partial u \partial v} \frac{\partial^{2} g}{\partial u \partial v}+\frac{\partial^{2} f}{\partial v^{2}} \frac{\partial^{2} g}{\partial u^{2}}, \\
\Delta^{2} h & =\frac{\partial^{4} h}{\partial u^{4}}+2 \frac{\partial^{4} h}{\partial u^{2} \partial v^{2}}+\frac{\partial^{4} h}{\partial v^{4}} .
\end{aligned}
$$

In this paper we study bifurcation of equilibrium forms of a thin round elastic plate lying on an elastic foundation under the action of a compressing

2000 Mathematics Subject Classification: 35Q72, 46T99.

Key words and phrases: bifurcation, elasticity, equilibrium form, Fredholm operator, variational gradient. 
force. Equilibrium forms of the plate may be found as solutions $w \in C_{0,0}^{4, \mu}(\bar{\Omega})$ and $\sigma \in C_{0,0}^{4, \mu}(\bar{\Omega})$ of the von Kármán equations

$$
\left\{\begin{array}{l}
\Delta^{2} w-[w, \sigma]+2 \alpha \Delta w+\beta w-\gamma w^{3}=0 \\
\Delta^{2} \sigma+\frac{1}{2}[w, w]=0
\end{array}\right.
$$

where $w(u, v)$ is a deflection function, $\sigma(u, v)$ is a stress function, $\alpha>0$ is the value of the compressing force, $\beta>0$ and $\gamma>0$ are parameters of the elastic foundation. We will suppose that $\gamma$ is a constant.

Numerous works have been devoted to the study of bifurcation in the von Kármán problems (see for instance [1], [6], [7], [8], [10], [11], [16], [18] and [21]). However, these investigations do not concern the situation when the plate is fixed to the elastic foundation. This case is very important in mechanics of elastic constructions. Physical models in which an elastic foundation appears have been studied only numerically for a long time. The investigation of such models by the use of modern bifurcation theory starts with [5] and [13]. It was noticed there that if we take into account additional nonlinear terms corresponding to the elastic base, then subcritical branches of solutions at a bifurcation point will occur. In this paper similar results will be obtained for the von Kármán equations (KE). We will show that both parameters appearing in (KE) influence bifurcation. For instance, if $\beta$ is larger than $\alpha^{2}$ there is no bifurcation, because the base is too hard and resists the compressing force. Moreover, the increase of $\beta$ (the hardness of the plate) may straighten the plate and may cause the appearance of subcritical branches of solutions.

The paper is organized as follows. In Section 2 we define a nonlinear map $F: X \times \mathbb{R}_{+}^{2} \rightarrow Y$, where

$$
X=C_{0,0}^{4, \mu}(\bar{\Omega}) \times C_{0,0}^{4, \mu}(\bar{\Omega}) \quad \text { and } \quad Y=C^{0, \mu}(\bar{\Omega}) \times C^{0, \mu}(\bar{\Omega}) .
$$

We reduce $(\mathrm{KE})$ to the operator equation

$$
F(x, p)=0,
$$

where $x=(w, \sigma)$ and $p=(\alpha, \beta)$. We prove that $F$ is a $C^{\infty}$ map with respect to all variables and it is a nonlinear operator of Fredholm type of index 0 with respect to $x$ in a neighbourhood of the point $(0, p)$ for each $p \in \mathbb{R}_{+}^{2}$. Finally, we show that solutions of (KE) are critical points of the $C^{\infty}$ functional $E: X \times \mathbb{R}_{+}^{2} \rightarrow \mathbb{R}$ given by

$$
\begin{aligned}
E(x, p)= & \frac{1}{2 \pi} \iint_{\Omega}\left((\Delta w)^{2}-(\Delta \sigma)^{2}-[w, w] \sigma\right) d u d v \\
& -\iint_{\Omega}\left(2 \alpha\left(\left(\frac{\partial w}{\partial u}\right)^{2}+\left(\frac{\partial w}{\partial v}\right)^{2}\right)-\beta w^{2}+\frac{1}{2} \gamma w^{4}\right) d u d v
\end{aligned}
$$


i.e. the map $F$ is a variational gradient for the functional $E$, and in further considerations we employ this fact. In Section 3 we prove the following necessary condition for bifurcation: if $(0, p)$ is a bifurcation point then $\operatorname{dim} \operatorname{Ker} F_{x}^{\prime}(0, p)>0$, where $F_{x}^{\prime}(0, p): X \rightarrow Y$ is the Fréchet derivative of $F$ with respect to $x$ at $(0, p)$. Section 4 contains a complete solution of the linearized problem

$$
F_{x}^{\prime}(0, p) h=0 .
$$

In Sections 5 and 6 we apply the Borisovich scheme for the study of bifurcation (see [4]). It was introduced during the investigation of bifurcation in the Plateau problem (see [2], [3]) and it is based on the analysis of the Lyapunov-Schmidt branching equation ([12], [20]) by the use of the Crandall-Rabinowitz bifurcation theorem ([9], [14]), the key function method due to Sapronov ([17], [18]) and the methods of the theory of singularities $([15])$.

Acknowledgments. The author wishes to express her thanks to Professor Czesław Szymczak from the Technical University of Gdańsk for suggesting the problem and to Professor Andrei Borisovich from the University of Gdańsk for several stimulating conversations. The author is also grateful to Professor Kazimierz Gęba from the Technical University of Gdańsk for his helpful comments and his interest in the publication of the paper.

2. Reduction to an operator equation. Let $F: X \times \mathbb{R}_{+}^{2} \rightarrow Y$ be given by

$$
F(x, p)=\left(F_{1}(x, p), F_{2}(x, p)\right),
$$

where $F_{1}, F_{2}: X \times \mathbb{R}_{+}^{2} \rightarrow C^{0, \mu}(\bar{\Omega})$ are defined as follows:

$$
\begin{aligned}
& F_{1}(x, p)=\Delta^{2} w-[w, \sigma]+2 \alpha \Delta w+\beta w-\gamma w^{3}, \\
& F_{2}(x, p)=-\Delta^{2} \sigma-\frac{1}{2}[w, w]
\end{aligned}
$$

for $x=(w, \sigma)$ and $p=(\alpha, \beta)$.

LEMMA 2.1. The pair $(x, p)$ belongs to the solution set of $(K E)$ if and only if $F(x, p)=0$.

The proof is immediate.

Definition 2.1. Let $X, Y$ be Banach spaces, let $U$ be a region in $X$ and let $n$ be a natural number. The map $F: X \rightarrow Y$ is said to be a nonlinear operator of Fredholm type of index $n$ in $U$ if

(i) $F$ is $C^{1}$,

(ii) for each $x \in U$ the Fréchet derivative $F^{\prime}(x): X \rightarrow Y$ is a Fredholm map of index $n$. 
If $F$ is $C^{1}$ and $F^{\prime}\left(x_{0}\right)$ is a Fredholm map of index $n$ for some $x_{0} \in X$, then $F$ is a nonlinear operator of Fredholm type of index $n$ in a neighbourhood of $x_{0}$. This is an obvious consequence of two facts: (1) the set $\Phi_{n}(X, Y)$ of Fredholm operators of index $n$ is open in $L(X, Y) ;(2)$ the Fréchet derivative $F^{\prime}: X \rightarrow L(X, Y)$ is continuous.

Theorem 2.2. The map $F: X \times \mathbb{R}_{+}^{2} \rightarrow Y$ given by (6)-(8) is $C^{\infty}$ with respect to all variables and for each $p \in \mathbb{R}_{+}^{2}$ it is a nonlinear operator of Fredholm type of index 0 with respect to $x$ in a neighbourhood of $(0, p)$, i.e. $F_{x}^{\prime}(0, p): X \rightarrow Y$ is a Fredholm map of index 0 .

Proof. The map $F$ is $C^{\infty}$, because all terms in $F_{1}, F_{2}$ are. Fix $p=$ $(\alpha, \beta) \in \mathbb{R}_{+}^{2}$. We now show that $F_{x}^{\prime}(0, p): X \rightarrow Y$ is a Fredholm map of index 0 . We have

$$
\begin{aligned}
& F_{x}^{\prime}(x, p) h \\
& \quad=\left(\Delta^{2} z-[z, \sigma]-[w, \eta]+2 \alpha \Delta z+\beta z-3 \gamma w^{2} z,-\Delta^{2} \eta-[w, z]\right) .
\end{aligned}
$$

Substituting $x_{0}=(0,0)$ into $(9)$ we get

$$
F_{x}^{\prime}(0, p) h=\left(\Delta^{2} z+2 \alpha \Delta z+\beta z,-\Delta^{2} \eta\right) .
$$

To prove that $F_{x}^{\prime}(0, p)$ is a Fredholm operator of index 0 , we only need to write it as a sum of an isomorphism and a completely continuous map. Note that

$$
F_{x}^{\prime}(0, p) h=A(h)+B(h),
$$

where $A, B: X \rightarrow Y$ are defined by

$$
A(z, \eta)=\left(\Delta^{2} z,-\Delta^{2} \eta\right), \quad B(z, \eta)=(2 \alpha \Delta z+\beta z, 0)=\left(B_{1}(z), 0\right) .
$$

It is well known that $\Delta$ is an isomorphism of $C_{0}^{m, \mu}(\bar{\Omega})$ onto $C^{m-2, \mu}(\bar{\Omega})$ for all $m \geq 2$. This implies that $\Delta^{2}: C_{0,0}^{4, \mu}(\bar{\Omega}) \rightarrow C^{0, \mu}(\bar{\Omega})$ is also an isomorphism, and so $A$ is an isomorphism of $X$ onto $Y$.

We now show that $B$ is completely continuous. To this end it suffices to show that $B_{1}: C_{0,0}^{4, \mu}(\bar{\Omega}) \rightarrow C^{0, \mu}(\bar{\Omega})$ is completely continuous. From the diagram

$$
C_{0,0}^{4, \mu}(\bar{\Omega}) \stackrel{J=I}{\longrightarrow} C_{0}^{2, \mu}(\bar{\Omega}) \stackrel{W=2 \alpha \Delta+\beta I}{\longrightarrow} C^{0, \mu}(\bar{\Omega})
$$

we obtain $B_{1}=W \circ J$. Now $W$ is a continuous map and $J$ is the natural completely continuous embedding, so $B_{1}$ is completely continuous.

The aim is now to show that the solution set of (KE) and the set of critical points of the functional $E$ given by (4) are the same.

Definition 2.2. Let $X, Y$ be Banach spaces continuously embedded in a Hilbert space $H$ with scalar product $(\cdot, \cdot)_{H}: H \times H \rightarrow \mathbb{R}$. Let $E: U \rightarrow \mathbb{R}$ be $C^{1}$ and $F: U \rightarrow Y$ be continuous, where $U$ is an open set in $X$. The 
map $F$ is called a variational gradient of the functional $E$ with respect to the scalar product in $H$ if

$$
E^{\prime}(x) h=(F(x), h)_{H}
$$

for each $x \in U$ and $h \in X$.

Fix $x \in U$. Let us remark that $E^{\prime}(x) h=0$ for each $h \in X$ if and only if $F(x)=0$. For this reason zeros of $F$ are called critical points of $E$.

Lemma 2.3. Let $c$ be a real number. Then

$$
\iint_{\Omega}(\Delta f+c f) g d u d v=\iint_{\Omega} f(\Delta g+c g) d u d v
$$

for all $f, g \in C_{0}^{2, \mu}(\bar{\Omega})$.

The equality (12) is checked at once, because $\Delta: C_{0}^{2, \mu}(\bar{\Omega}) \rightarrow C^{0, \mu}(\bar{\Omega})$ is self-adjoint with respect to the inner product in $L^{2}(\Omega)$.

Let $H=L^{2}(\Omega) \times L^{2}(\Omega)$. It is well known that $H$ with the standard scalar product defined by

$$
\left(\left(z_{1}, \eta_{1}\right),\left(z_{2}, \eta_{2}\right)\right)_{H}=\frac{1}{\pi} \iint_{\Omega}\left(z_{1} z_{2}+\eta_{1} \eta_{2}\right) d u d v
$$

is a Hilbert space. Moreover, $X=C_{0,0}^{4, \mu}(\bar{\Omega}) \times C_{0,0}^{4, \mu}(\bar{\Omega})$ and $Y=C^{0, \mu}(\bar{\Omega}) \times$ $C^{0, \mu}(\bar{\Omega})$ are continuously embedded in $H$.

TheOREM 2.4. For each $p \in \mathbb{R}_{+}^{2}$ the map $F(\cdot, p): X \rightarrow Y$ given by (6)-(8) is a variational gradient of the functional $E(\cdot, p): X \rightarrow \mathbb{R}$ defined by (4) with respect to the scalar product (13), i.e.

$$
E_{x}^{\prime}(x, p) h=(F(x, p), h)_{H}
$$

for all $x, h \in X$.

Proof. Let $p=(\alpha, \beta) \in \mathbb{R}_{+}^{2}$. Since $E(\cdot, p): X \rightarrow \mathbb{R}$ is $C^{\infty}$, we get

$$
E_{x}^{\prime}(x, p) h=\left.\frac{d}{d t} E(x+t h, p)\right|_{t=0}
$$

for all $x, h \in X$. Fix $x=(w, \sigma), h=(z, \eta)$ in $X$. An easy computation shows that

$$
\begin{aligned}
\left.\frac{d}{d t} E(x+t h, p)\right|_{t=0}= & \frac{1}{\pi} \iint_{\Omega}(\Delta w \Delta z-\Delta \sigma \Delta \eta-\sigma[w, z]) d u d v \\
& -\frac{1}{\pi} \iint_{\Omega} \frac{1}{2}[w, w] \eta+2 \alpha\left(\frac{\partial w}{\partial u} \frac{\partial z}{\partial u}+\frac{\partial w}{\partial v} \frac{\partial z}{\partial v}\right) d u d v \\
& +\frac{1}{\pi} \iint_{\Omega}\left(\beta w z-\gamma w^{3} z\right) d u d v
\end{aligned}
$$


Using Lemma 2.3 we get

$$
\begin{aligned}
& \iint_{\Omega} \Delta w \Delta z d u d v=\iint_{\Omega}\left(\Delta^{2} w\right) z d u d v \\
& \iint_{\Omega} \Delta \sigma \Delta \eta d u d v=\iint_{\Omega}\left(\Delta^{2} \sigma\right) \eta d u d v .
\end{aligned}
$$

We conclude from the definition of $F$ that to finish the proof it remains to show the equalities

$$
\iint_{\Omega} \sigma[w, z] d u d v=\iint_{\Omega}[w, \sigma] z d u d v
$$

and

$$
\iint_{\Omega}\left(\frac{\partial w}{\partial u} \frac{\partial z}{\partial u}+\frac{\partial w}{\partial v} \frac{\partial z}{\partial v}\right) d u d v=-\iint_{\Omega}(\Delta w) z d u d v
$$

In order to get these equalities, we decompose the integrals on the left-hand side into appropriate terms and integrate by parts. The details are left to the reader.

3. A necessary condition for bifurcation. Let $\Gamma \subset X \times \mathbb{R}_{+}^{2}$ be given by $\Gamma=\left\{(0, p): p \in \mathbb{R}_{+}^{2}\right\}$. The set $\Gamma$ is called the trivial family (branch) of solutions of equation (3). In this section we prove a necessary condition for bifurcation at the points of $\Gamma$.

Definition 3.1. A point $\left(0, p_{0}\right) \in \Gamma$ is said to be a bifurcation point of equation (3) if in every neighbourhood of $\left(0, p_{0}\right)$ there exists a point $(x, p) \in X \times \mathbb{R}_{+}^{2}$ such that $x \neq 0$ and $F(x, p)=0$.

THEOREM 3.1. If $\left(0, p_{0}\right) \in \Gamma$ is a bifurcation point of equation (3), then $\operatorname{dim} \operatorname{Ker} F_{x}^{\prime}\left(0, p_{0}\right)>0$.

Proof. Suppose, contrary to our claim, that $\operatorname{dim} \operatorname{Ker} F_{x}^{\prime}\left(0, p_{0}\right)=0$. Since $F_{x}^{\prime}\left(0, p_{0}\right): X \rightarrow Y$ is a Fredholm map of index 0 , we have

$$
\operatorname{dim} \operatorname{Ker} F_{x}^{\prime}\left(0, p_{0}\right)=\operatorname{codim} \operatorname{Im} F_{x}^{\prime}\left(0, p_{0}\right)
$$

hence $Y=\operatorname{Im} F_{x}^{\prime}\left(0, p_{0}\right)$, and so $F_{x}^{\prime}\left(0, p_{0}\right)$ is an isomorphism. Applying the implicit function theorem we see that in a small neighbourhood of $\left(0, p_{0}\right)$ the solution set of (3) is contained in $\Gamma$.

4. The linearized problem. The aim of this section is to find all critical values of the parameters $\alpha$ and $\beta$.

Definition 4.1. Let $p=(\alpha, \beta)$. The numbers $\alpha$ and $\beta$ are called critical values of parameters if the necessary condition for bifurcation at the point $(0, p)$, stated in Theorem 3.1, is satisfied. 
To check the necessary condition for bifurcation, we note that $\operatorname{Ker} F_{x}^{\prime}(0, p)$ $=\operatorname{Ker}\left(\Delta^{2}+2 \alpha \Delta+\beta I\right) \times \operatorname{Ker}\left(\Delta^{2}\right)$ by (10). Since $\Delta^{2}: C_{0,0}^{4, \mu}(\bar{\Omega}) \rightarrow C^{0, \mu}(\bar{\Omega})$ is an isomorphism, we have $\operatorname{Ker} F_{x}^{\prime}(0, p)=\operatorname{Ker}\left(\Delta^{2}+2 \alpha \Delta+\beta I\right) \times\{0\}$. From now on, to simplify notation, we write $N(p)$ instead of $\operatorname{Ker} F_{x}^{\prime}(0, p)$.

Define $\delta=\alpha^{2}-\beta$. If $\delta \geq 0$ then $a=-\alpha-\sqrt{\delta}$ and $b=-\alpha+\sqrt{\delta}$. For every nonnegative integer $k$ let $J_{k}: \mathbb{R} \rightarrow \mathbb{R}$ be the Bessel function given by

$$
J_{k}(s)=\frac{1}{\pi} \int_{0}^{\pi} \cos (s \sin t+k t) d t .
$$

Let $(r, \varphi)$ denote the polar coordinates of a point $(u, v) \in \bar{\Omega}$.

We give a complete solution of (5) in Table 1.

Table 1

\begin{tabular}{|c|c|c|c|c|}
\hline \multicolumn{3}{|c|}{ Assumptions } & \multicolumn{2}{|r|}{ Results } \\
\hline$\delta$ & & and $b$ & $\operatorname{dim} N(p)$ & Base of $N(p)$ \\
\hline- & & lefined & 0 & does not exist \\
\hline $\begin{array}{l}+ \\
\text { or } 0\end{array}$ & $\forall_{k \geq 0}$ & $\begin{array}{l}J_{k}(a) \neq 0 \\
J_{k}(b) \neq 0\end{array}$ & 0 & does not exist \\
\hline 0 & & $J_{0}(a)=0$ & 1 & $f_{1}(u, v)=\left(J_{0}(a r), 0\right)$ \\
\hline+ & $\forall_{k \geq 0}$ & $\begin{array}{l}J_{k}(b) \neq 0 \\
J_{0}(a)=0\end{array}$ & 1 & $f_{1}(u, v)=\left(J_{0}(a r), 0\right)$ \\
\hline+ & $\forall_{k \geq 0}$ & $\begin{array}{l}J_{k}(a) \neq 0 \\
J_{0}(b)=0\end{array}$ & 1 & $f_{1}(u, v)=\left(J_{0}(b r), 0\right)$ \\
\hline 0 & $\exists_{k>0}$ & $J_{k}(a)=0$ & 2 & $\begin{array}{l}f_{1}(u, v)=\left(J_{k}(a r) \cos (k \varphi), 0\right) \\
f_{2}(u, v)=\left(J_{k}(a r) \sin (k \varphi), 0\right)\end{array}$ \\
\hline+ & & $\begin{array}{l}J_{0}(a)=0 \\
J_{0}(b)=0\end{array}$ & 2 & $\begin{array}{l}f_{1}(u, v)=\left(J_{0}(a r), 0\right) \\
f_{2}(u, v)=\left(J_{0}(b r), 0\right)\end{array}$ \\
\hline+ & $\begin{array}{l}\forall_{l \geq 0} \\
\exists_{k>0}\end{array}$ & $\begin{array}{l}J_{l}(b) \neq 0 \\
J_{k}(a)=0\end{array}$ & 2 & $\begin{array}{l}f_{1}(u, v)=\left(J_{k}(a r) \cos (k \varphi), 0\right) \\
f_{2}(u, v)=\left(J_{k}(a r) \sin (k \varphi), 0\right)\end{array}$ \\
\hline+ & $\begin{array}{l}\forall_{k \geq 0} \\
\exists_{l>0}\end{array}$ & $\begin{array}{l}J_{k}(a) \neq 0 \\
J_{l}(b)=0\end{array}$ & 2 & $\begin{array}{l}f_{1}(u, v)=\left(J_{l}(b r) \cos (l \varphi), 0\right) \\
f_{2}(u, v)=\left(J_{l}(b r) \sin (l \varphi), 0\right)\end{array}$ \\
\hline+ & $\exists_{k>0}$ & $\begin{array}{l}J_{k}(a)=0 \\
J_{0}(b)=0\end{array}$ & 3 & $\begin{array}{l}f_{1}(u, v)=\left(J_{k}(a r) \cos (k \varphi), 0\right) \\
f_{2}(u, v)=\left(J_{k}(a r) \sin (k \varphi), 0\right) \\
f_{3}(u, v)=\left(J_{0}(b r), 0\right)\end{array}$ \\
\hline+ & $\exists_{k>0}$ & $\begin{array}{l}J_{k}(b)=0 \\
J_{0}(a)=0\end{array}$ & 3 & $\begin{array}{l}f_{1}(u, v)=\left(J_{0}(a r), 0\right) \\
f_{2}(u, v)=\left(J_{k}(b r) \cos (k \varphi), 0\right) \\
f_{3}(u, v)=\left(J_{k}(b r) \sin (k \varphi), 0\right)\end{array}$ \\
\hline+ & $\exists_{k, l>l}$ & $\begin{aligned} J_{k}(a) & =0 \\
J_{l}(b) & =0\end{aligned}$ & 4 & $\begin{array}{l}f_{1}(u, v)=\left(J_{k}(a r) \cos (k \varphi), 0\right) \\
f_{2}(u, v)=\left(J_{k}(a r) \sin (k \varphi), 0\right) \\
f_{3}(u, v)=\left(J_{l}(b r) \cos (l \varphi), 0\right) \\
f_{4}(u, v)=\left(J_{l}(b r) \sin (l \varphi), 0\right)\end{array}$ \\
\hline
\end{tabular}

We divide the proof of the results from Table 1 into a sequence of lemmas. 
Lemma 4.1. If $\delta<0$, then $\operatorname{Ker}\left(\Delta^{2}+2 \alpha \Delta+\beta I\right)=\{0\}$.

Proof. Let $z \in C_{0,0}^{4, \mu}(\bar{\Omega})$. Then $\Delta z \in C_{0}^{2, \mu}(\bar{\Omega})$. Using Lemma 2.3 we get

$$
\iint_{\Omega}\left(\Delta^{2} z\right) z d u d v=\iint_{\Omega}(\Delta z) \Delta z d u d v=\iint_{\Omega}(\Delta z)^{2} d u d v
$$

and hence

$$
\begin{aligned}
& \iint_{\Omega}\left(\Delta^{2} z+2 \alpha \Delta z+\beta z\right) z d u d v \\
& \quad=\iint_{\Omega}\left(\Delta^{2} z\right) z d u d v+\iint_{\Omega} 2 \alpha(\Delta z) z d u d v+\iint_{\Omega} \beta z^{2} d u d v \\
& =\iint_{\Omega}(\Delta z)^{2} d u d v+\iint_{\Omega} 2 \alpha(\Delta z) z d u d v+\iint_{\Omega} \beta z^{2} d u d v \\
& =\iint_{\Omega}\left((\Delta z)^{2}+2 \alpha(\Delta z) z+\alpha^{2} z^{2}\right) d u d v+\iint_{\Omega}\left(\beta-\alpha^{2}\right) z^{2} d u d v \\
& =\iint_{\Omega}(\Delta z+\alpha z)^{2} d u d v+\iint_{\Omega}\left(\beta-\alpha^{2}\right) z^{2} d u d v .
\end{aligned}
$$

Now, let $z \in \operatorname{Ker}\left(\Delta^{2}+2 \alpha \Delta+\beta I\right)$. Then $\iint_{\Omega}\left(\Delta^{2} z+2 \alpha \Delta z+\beta z\right) z d u d v=0$. Applying (15) we get

$$
\iint_{\Omega}(\Delta z+\alpha z)^{2} d u d v+\iint_{\Omega}\left(\beta-\alpha^{2}\right) z^{2} d u d v=0
$$

Since $\delta<0$, we have $\beta-\alpha^{2}>0$. Therefore (16) yields $z=0$ on $\bar{\Omega}$, which completes the proof.

LEMMA 4.2. Let $\Delta+\alpha I: C_{0}^{2, \mu}(\bar{\Omega}) \rightarrow C^{0, \mu}(\bar{\Omega})$. If $\delta=0$, then

$$
\operatorname{Ker}\left(\Delta^{2}+2 \alpha \Delta+\beta I\right)=\operatorname{Ker}(\Delta+\alpha I) .
$$

Proof. Choose $z \in C_{0,0}^{4, \mu}(\bar{\Omega})$. Then $\Delta z+\alpha z \in C_{0}^{2, \mu}(\bar{\Omega})$. Applying Lemma 2.3 we get

$$
\begin{aligned}
\iint_{\Omega}(\Delta z+\alpha z)(\Delta z+\alpha z) d u d v & =\iint_{\Omega}(\Delta(\Delta z+\alpha z)+\alpha(\Delta z+\alpha z)) z d u d v \\
& =\iint_{\Omega}\left(\Delta^{2} z+2 \alpha \Delta z+\alpha^{2} z\right) z d u d v \\
& =\iint_{\Omega}\left(\Delta^{2} z+2 \alpha \Delta z+\beta z\right) z d u d v .
\end{aligned}
$$

Hence if $z \in \operatorname{Ker}\left(\Delta^{2}+2 \alpha \Delta+\beta I\right)$, then $z \in \operatorname{Ker}(\Delta+\alpha I)$.

On the other hand $\operatorname{Ker}(\Delta+\alpha I) \subset C_{0}^{\infty}(\bar{\Omega})$. Therefore if $z \in \operatorname{Ker}(\Delta+\alpha I)$, then $z \in C_{0,0}^{4, \mu}(\bar{\Omega})$, and so

$$
\left(\Delta^{2}+2 \alpha \Delta+\beta I\right)(z)=(\Delta+\alpha I) \circ(\Delta+\alpha I)(z)=0 .
$$


Lemma 4.3. Suppose that $\delta>0$. Let $H_{1}, H_{2}: C_{0}^{2, \mu}(\bar{\Omega}) \rightarrow C^{0, \mu}(\bar{\Omega})$ be given by

$$
H_{1}(z)=\Delta z-a z, \quad H_{2}(z)=\Delta z-b z .
$$

Then

$$
\operatorname{Ker}\left(\Delta^{2}+2 \alpha \Delta+\beta I\right)=\operatorname{Ker}(\Delta-a I) \oplus \operatorname{Ker}(\Delta-b I) .
$$

Proof. We denote the operator $\Delta^{2}+2 \alpha \Delta+\beta I: C_{0,0}^{4, \mu}(\bar{\Omega}) \rightarrow C^{0, \mu}(\bar{\Omega})$ briefly by $G$. We have to prove that

$\operatorname{Ker} G=\operatorname{Ker} H_{1} \oplus \operatorname{Ker} H_{2}$.

First note that $\operatorname{Ker} H_{i} \subset C_{0}^{\infty}(\bar{\Omega}) \subset C_{0,0}^{4, \mu}(\bar{\Omega})$ and it is easy to check that

Ker $H_{1} \cap \operatorname{Ker} H_{2}=\{0\}$,

so it suffices to show that $\operatorname{Ker} G=\operatorname{Ker} H_{1}+\operatorname{Ker} H_{2}$. For $z \in C_{0,0}^{4, \mu}(\bar{\Omega})$, we have

$$
\begin{aligned}
H_{1} \circ H_{2}(z) & =(\Delta-a I) \circ(\Delta-b I)(z) \\
& =(\Delta-a I)(\Delta z)-(\Delta-a I)(b z) \\
& =\Delta^{2} z-a \Delta z-b \Delta z+a b z=\Delta^{2} z+2 \alpha \Delta z+\beta z=G(z)
\end{aligned}
$$

and similarly

$$
H_{2} \circ H_{1}(z)=G(z) .
$$

Hence $\operatorname{Ker} H_{i} \subset \operatorname{Ker} G$ and so $\operatorname{Ker} H_{1}+\operatorname{Ker} H_{2} \subset \operatorname{Ker} G$. To prove the opposite inclusion, let $z \in \operatorname{Ker} G$. By (17) and (18),

$$
x_{1}=H_{1}(z) \in \operatorname{Ker} H_{2} \quad \text { and } \quad x_{2}=H_{2}(z) \in \operatorname{Ker} H_{1} .
$$

Therefore

$$
x_{1}-x_{2}=H_{1}(z)-H_{2}(z)=(\Delta-a I)(z)-(\Delta-b I)(z)=(b-a) z .
$$

Since $b-a \neq 0$, the proof is complete.

Summary. It is known that for $m \geq 2, \lambda \in \mathbb{R} \backslash\{0\}$ is an eigenvalue of $\Delta: C_{0}^{m, \mu}(\bar{\Omega}) \rightarrow C^{m-2, \mu}(\bar{\Omega})$ if and only if $\lambda$ is a zero of one of the Bessel functions $J_{k}$ defined by (14). If $k=0$, then $\operatorname{dim}(\Delta-\lambda I)=1$ and $\operatorname{Ker}(\Delta-\lambda I)$ is generated by the function $f(u, v)=J_{0}(\lambda r)$. If $k \geq 1$, then $\operatorname{dim}(\Delta-\lambda I)=2$ and $\operatorname{Ker}(\Delta-\lambda I)$ is generated by two linearly independent functions: $f(u, v)=J_{k}(\lambda r) \cos (k \varphi)$ and $g(u, v)=J_{k}(\lambda r) \sin (k \varphi)$.

Combining Lemmas 4.1-4.3 with the properties of eigenspaces of the Laplace operator we obtain the results given in Table 1.

5. Bifurcation at simple points of $\Gamma$. In this section we investigate bifurcation at a simple point of $\Gamma$, i.e. at a point $\left(0, p_{0}\right) \in \Gamma$ such that $\operatorname{dim} N\left(p_{0}\right)=1$. We use the scheme introduced by A. Borisovich (see [2] and [4]) and based on the Crandall-Rabinowitz theorem on simple bifurcation points (see [9] and [14]). 
TheOREm 5.1. Let $X, Y$ be real Banach spaces continuously embedded in a real Hilbert space $H$ with scalar product $(\cdot, \cdot)_{H}: H \times H \rightarrow \mathbb{R}$ and let $E: X_{\varepsilon}\left(x_{0}\right) \times \mathbb{R}_{\varepsilon}\left(\lambda_{0}\right) \rightarrow \mathbb{R}$ be a $C^{r+1}$ functional, $r \geq 3$. Consider the equation

$$
F(x, \lambda)=0
$$

with a real parameter $\lambda$, where $F: X_{\varepsilon}\left(x_{0}\right) \times \mathbb{R}_{\varepsilon}\left(\lambda_{0}\right) \rightarrow Y$ is a $C^{r}$ map. Assume that:

1. $F\left(x_{0}, \lambda\right)=0$ for every $\lambda \in \mathbb{R}_{\varepsilon}\left(\lambda_{0}\right)$.

2. $\operatorname{dim} \operatorname{Ker} F_{x}^{\prime}\left(x_{0}, \lambda_{0}\right)=1, F_{x}^{\prime}\left(x_{0}, \lambda_{0}\right) e=0,(e, e)_{H}=1$.

3. codim $\operatorname{Im} F_{x}^{\prime}\left(x_{0}, \lambda_{0}\right)=1$.

4. $E_{x}^{\prime}(x, \lambda) h=(F(x, \lambda), h)_{H}$ for all $(x, \lambda) \in X_{\varepsilon}\left(x_{0}\right) \times \mathbb{R}_{\varepsilon}\left(\lambda_{0}\right)$ and for each $h \in X$.

5. $E_{x x \lambda}^{\prime \prime \prime}\left(x_{0}, \lambda_{0}\right) e e \neq 0$.

Then $\left(x_{0}, \lambda_{0}\right)$ is a bifurcation point of the equation $F(x, \lambda)=0$ and the solution set of this equation in a small neighbourhood of $\left(x_{0}, \lambda_{0}\right)$ is the union of the trivial family $\Gamma_{1}=\left\{\left(x_{0}, \lambda\right): \lambda \in \mathbb{R}_{\varepsilon}\left(\lambda_{0}\right)\right\}$ and a $C^{r-2}$ curve $\Gamma_{2}$ which intersects $\Gamma_{1}$ at $\left(x_{0}, \lambda_{0}\right)$ only and is given parametrically as follows:

$$
\Gamma_{2}=\{(x(t), \lambda(t)):|t|<\varsigma\}
$$

where $x(0)=x_{0}, \lambda(0)=\lambda_{0}$ and $x^{\prime}(0)=e$.

The symbols $X_{\varepsilon}\left(x_{0}\right), \mathbb{R}_{\varepsilon}\left(\lambda_{0}\right)$ denote small neighbourhoods of $x_{0}, \lambda_{0}$, respectively, i.e. $X_{\varepsilon}\left(x_{0}\right)=\left\{x \in X:\left\|x-x_{0}\right\|<\varepsilon\right\}, \mathbb{R}_{\varepsilon}\left(\lambda_{0}\right)=\left(\lambda_{0}-\varepsilon, \lambda_{0}+\varepsilon\right)$.

From now on, as in Section 2, we denote by $H$ the Hilbert space $L^{2}(\Omega) \times$ $L^{2}(\Omega)$ with the scalar product $(\cdot, \cdot)_{H}: H \times H \rightarrow \mathbb{R}$ given by (13).

TheOREM 5.2 (bifurcation with respect to $\alpha$ ). Let $p_{0}=\left(\alpha_{0}, \beta_{0}\right) \in \mathbb{R}_{+}^{2}$ satisfy

$$
\operatorname{dim} N\left(p_{0}\right)=1, \quad F_{x}^{\prime}\left(0, p_{0}\right) e=0, \quad(e, e)_{H}=1, \quad e=\left(e_{1}, 0\right) .
$$

Then $\left(0, \alpha_{0}\right) \in X \times \mathbb{R}_{+}$is a bifurcation point of the equation

$$
F\left(x, \alpha, \beta_{0}\right)=0
$$

and the solution set of (20) in a small neighbourhood of $\left(0, \alpha_{0}\right)$ is the union of $\Gamma_{1}=\left\{(0, \alpha): \alpha \in \mathbb{R}_{+}\right\}$and a $C^{\infty}$ curve $\Gamma_{2}$ which intersects $\Gamma_{1}$ at $\left(0, \alpha_{0}\right)$ only and is given parametrically as follows:

$$
\Gamma_{2}=\{(x(t), \alpha(t)):|t|<\varsigma\},
$$

where $x(0)=0, \alpha(0)=\alpha_{0}$ and $x^{\prime}(0)=e$.

Proof. Since $\alpha_{0}>0$, there exists $\varepsilon>0$ such that $\mathbb{R}_{\varepsilon}\left(\alpha_{0}\right) \subset \mathbb{R}_{+}$. We check that the operator $F\left(\cdot, \cdot, \beta_{0}\right): X_{\varepsilon}(0) \times \mathbb{R}_{\varepsilon}\left(\alpha_{0}\right) \rightarrow Y$ satisfies the assumptions of Theorem 5.1. 
Obviously, $F\left(0, \alpha, \beta_{0}\right)=0$ for each $\alpha \in \mathbb{R}_{\varepsilon}\left(\alpha_{0}\right)$. From Theorem 2.2 we know that $F\left(\cdot, \alpha_{0}, \beta_{0}\right): X \rightarrow Y$ is $C^{\infty}$ and

$$
\operatorname{dim} N\left(p_{0}\right)=\operatorname{codim} \operatorname{Im} F_{x}^{\prime}\left(0, \alpha_{0}, \beta_{0}\right) .
$$

Combining this with (19) we get

$$
\operatorname{codim} \operatorname{Im} F_{x}^{\prime}\left(0, \alpha_{0}, \beta_{0}\right)=1 .
$$

By Theorem 2.4,

$$
E_{x}^{\prime}\left(x, \alpha, \beta_{0}\right) h=\left(F\left(x, \alpha, \beta_{0}\right), h\right)_{H}
$$

for all $x, h \in X$ and $\alpha \in \mathbb{R}_{+}$. The spaces $X, Y$ are continuously embedded in $H$. Therefore differentiating with respect to $x$ we have

$$
E_{x x}^{\prime \prime}\left(x, \alpha, \beta_{0}\right) h g=\left(F_{x}^{\prime}\left(x, \alpha, \beta_{0}\right) h, g\right)_{H}
$$

for each $\alpha \in \mathbb{R}_{+}$and for all $x, h, g \in X$. Using (9) we obtain

$E_{x x}^{\prime \prime}\left(x, \alpha, \beta_{0}\right) h g=\frac{1}{\pi} \iint_{\Omega}\left(-\Delta^{2} \eta-[w, z]\right) \eta_{1} d u d v$

$$
+\frac{1}{\pi} \iint_{\Omega}\left(\Delta^{2} z-[z, \sigma]-[w, \eta]+2 \alpha \Delta z+\beta_{0} z-3 \gamma w^{2} z\right) z_{1} d u d v,
$$

where $x=(w, \sigma), h=(z, \eta), g=\left(z_{1}, \eta_{1}\right)$. Hence we get at once

$$
E_{x x \alpha}^{\prime \prime \prime}\left(x, \alpha, \beta_{0}\right) h g=\frac{1}{\pi} \iint_{\Omega} 2(\Delta z) z_{1} d u d v .
$$

Substituting $x=(0,0), \alpha=\alpha_{0}$ and $h=g=e$ we have

$$
E_{x x \alpha}^{\prime \prime \prime}\left(0, \alpha_{0}, \beta_{0}\right) e e=\frac{1}{\pi} \iint_{\Omega} 2\left(\Delta e_{1}\right) e_{1} d u d v .
$$

We have to consider two cases.

CAse 1: $\alpha_{0}^{2}=\beta_{0}$. By Lemma 4.2, $\Delta e_{1}+\alpha_{0} e_{1}=0$. Hence

$$
E_{x x \alpha}^{\prime \prime \prime}\left(0, \alpha_{0}, \beta_{0}\right) e e=-\frac{2 \alpha_{0}}{\pi} \iint_{\Omega} e_{1}^{2} d u d v=-2 \alpha_{0}(e, e)_{H}=-2 \alpha_{0}<0 .
$$

CASE 2: $\delta_{0}=\alpha_{0}^{2}-\beta_{0}>0$. We conclude from Lemma 4.3 that either $\Delta e_{1}-a_{0} e_{1}=0$ or $\Delta e_{1}-b_{0} e_{1}=0$, where $a_{0}=-\alpha_{0}-\sqrt{\delta_{0}}, b_{0}=-\alpha_{0}+\sqrt{\delta_{0}}$. It is evident that $a_{0}<0$ and $b_{0}<0$. Suppose that $\Delta e_{1}-a_{0} e_{1}=0$. Then

$$
E_{x x \alpha}^{\prime \prime \prime}\left(0, \alpha_{0}, \beta_{0}\right) e e=\frac{2 a_{0}}{\pi} \iint_{\Omega} e_{1}^{2} d u d v=2 a_{0}(e, e)_{H}=2 a_{0}<0,
$$

and similarly for $\Delta e_{1}-b_{0} e_{1}=0$. In this way we have established that assumptions 1-5 of Theorem 5.1 hold, which completes the proof.

TheOREM 5.3 (bifurcation with respect to $\beta$ ). Let $p_{0}=\left(\alpha_{0}, \beta_{0}\right) \in \mathbb{R}_{+}^{2}$ satisfy condition (19). Then $\left(0, \beta_{0}\right) \in X \times \mathbb{R}_{+}$is a bifurcation point of the 
equation

$$
F\left(x, \alpha_{0}, \beta\right)=0
$$

and the solution set of $(21)$ in a small neighbourhood of $\left(0, \beta_{0}\right)$ is the union of $\widehat{\Gamma}_{1}=\left\{(0, \beta): \beta \in \mathbb{R}_{+}\right\}$and a $C^{\infty}$ curve $\widehat{\Gamma}_{2}$ which intersects $\widehat{\Gamma}_{1}$ at $\left(0, \beta_{0}\right)$ only and is given parametrically as follows:

$$
\widehat{\Gamma}_{2}=\{(\widehat{x}(t), \beta(t)):|t|<\varsigma\},
$$

where $\widehat{x}(0)=0, \beta(0)=\beta_{0}$ and $\widehat{x}^{\prime}(0)=e$.

Proof. We again apply Theorem 5.1. Since $\beta_{0}>0$, there exists $\varepsilon>0$ such that $\mathbb{R}_{\varepsilon}\left(\beta_{0}\right) \subset \mathbb{R}_{+}$. Analysis similar to that in the proof of Theorem 5.2 shows that the map $F\left(\cdot, \alpha_{0}, \cdot\right): X_{\varepsilon}(0) \times \mathbb{R}_{\varepsilon}\left(\beta_{0}\right) \rightarrow Y$ and the functional $E\left(\cdot, \alpha_{0}, \cdot\right): X_{\varepsilon}(0) \times \mathbb{R}_{\varepsilon}\left(\beta_{0}\right) \rightarrow \mathbb{R}$ satisfy assumptions $1-4$ of Theorem 5.1. Therefore it remains to check assumption 5. By Theorem 2.4 we have

$$
E_{x x}^{\prime \prime}\left(x, \alpha_{0}, \beta\right) h g=\left(F_{x}^{\prime}\left(x, \alpha_{0}, \beta\right) h, g\right)_{H}
$$

for each $\beta \in \mathbb{R}_{+}$and for all $x, h, g \in X$. Using (9) we get

$$
\begin{aligned}
E_{x x}^{\prime \prime}\left(x, \alpha_{0}, \beta\right) h g & =\frac{1}{\pi} \iint_{\Omega}\left(-\Delta^{2} \eta-[w, z]\right) \eta_{1} d u d v \\
& +\frac{1}{\pi} \iint_{\Omega}\left(\Delta^{2} z-[z, \sigma]-[w, \eta]+2 \alpha_{0} \Delta z+\beta z-3 \gamma w^{2} z\right) z_{1} d u d v
\end{aligned}
$$

where $x=(w, \sigma), h=(z, \eta), g=\left(z_{1}, \eta_{1}\right)$. Thus

$$
E_{x x \beta}^{\prime \prime \prime}\left(x, \alpha_{0}, \beta\right) h g=\frac{1}{\pi} \iint_{\Omega} z z_{1} d u d v .
$$

Substituting $x=(0,0), \beta=\beta_{0}$ and $h=g=e$ we obtain

$$
E_{x x \beta}^{\prime \prime \prime}\left(0, \alpha_{0}, \beta_{0}\right) e e=\frac{1}{\pi} \iint_{\Omega} e_{1}^{2} d u d v=(e, e)_{H}=1>0,
$$

which completes the proof.

6. The shape of bifurcation branches. Let $\left(0, p_{0}\right) \in X \times \mathbb{R}_{+}^{2}$ be a fixed point satisfying condition (19). We now study the changes in geometrical structure of the solution set of $F(x, p)=0$ near $\left(0, p_{0}\right)$. We use the scheme based on the Lyapunov-Schmidt finite-dimensional reduction, adapted from [4]. The theorem below describes it.

THEOREM 6.1. Let $X, Y$ be real Banach spaces continuously embedded in a real Hilbert space $H$ with inner product $(\cdot, \cdot)_{H}: H \times H \rightarrow \mathbb{R}$ and let $E: X_{\varepsilon}\left(x_{0}\right) \times \mathbb{R}_{\varepsilon}\left(\lambda_{0}\right) \rightarrow \mathbb{R}$ be a $C^{r+1}$ functional, $r \geq 3$. Consider the equation

$$
F(x, \lambda)=0,
$$


where $F: X_{\varepsilon}\left(x_{0}\right) \times \mathbb{R}_{\varepsilon}\left(\lambda_{0}\right) \rightarrow Y$ is a $C^{r}$ mapping. Assume that conditions 1-5 of Theorem 5.1 hold and define

$$
\begin{aligned}
& C_{1}=E_{x x \lambda}^{\prime \prime \prime}\left(x_{0}, \lambda_{0}\right) e e, \\
& C_{2}=E_{x x x}^{\prime \prime \prime}\left(x_{0}, \lambda_{0}\right) e e e, \\
& C_{3}=E_{x x x x}^{(4)}\left(x_{0}, \lambda_{0}\right) \text { eeee }+3 E_{x x x}^{\prime \prime \prime}\left(x_{0}, \lambda_{0}\right) e e y_{0},
\end{aligned}
$$

where $y_{0}$ is a unique solution of the linear equation

$$
F_{x}^{\prime}\left(x_{0}, \lambda_{0}\right) y_{0}+\left(y_{0}, e\right)_{H} e=-F_{x x}^{\prime \prime}\left(x_{0}, \lambda_{0}\right) e e .
$$

Under the above assumptions:

(i) If $C_{1} \neq 0$ then $\left(x_{0}, \lambda_{0}\right)$ is a bifurcation point of the equation (23) and the solution set of $(23)$ in a small neighbourhood of $\left(x_{0}, \lambda_{0}\right)$ is the union of the trivial family

$$
\Gamma_{1}: \quad x_{1}(\lambda)=x_{0}, \quad \lambda \in \mathbb{R}_{\varepsilon}\left(\lambda_{0}\right),
$$

and a $C^{r-2}$ family $\Gamma_{2}$ which intersects $\Gamma_{1}$ at $\left(x_{0}, \lambda_{0}\right)$ only.

(ii) If $C_{1} \neq 0$ and $C_{2} \neq 0$ then $\Gamma_{2}$ can be parametrized as follows:

$$
\Gamma_{2}: \quad x_{2}(\lambda)=x_{0}+D_{2}\left(\lambda-\lambda_{0}\right) e+o\left(\left|\lambda-\lambda_{0}\right|\right), \quad \lambda \in \mathbb{R}_{\varepsilon}\left(\lambda_{0}\right),
$$

where $D_{2}=-2 C_{2} / C_{1}$, and we have transcritical branching at $\left(x_{0}, \lambda_{0}\right)$.

(iii) If $C_{1} \neq 0, C_{2}=0$ and $C_{3} \neq 0$ then the parametrization of $\Gamma_{2}$ depends on the signs of $C_{1}$ and $C_{3}$.

If $C_{1} C_{3}>0$ then

$\Gamma_{2}: \quad x_{2}^{ \pm}(\lambda)=x_{0} \pm \sqrt{\left|D_{3}\right|}\left(\lambda_{0}-\lambda\right)^{1 / 2} e+o\left(\left|\lambda-\lambda_{0}\right|^{1 / 2}\right), \quad \lambda \in\left(\lambda_{0}-\varepsilon, \lambda_{0}\right]$, and we have subcritical branching at $\left(x_{0}, \lambda_{0}\right)$.

If $C_{1} C_{3}<0$ then

$\Gamma_{2}: \quad x_{2}^{ \pm}(\lambda)=x_{0} \pm \sqrt{D_{3}}\left(\lambda-\lambda_{0}\right)^{1 / 2} e+o\left(\left|\lambda-\lambda_{0}\right|^{1 / 2}\right), \quad \lambda \in\left[\lambda_{0}, \lambda_{0}+\varepsilon\right)$, and we have postcritical branching at $\left(x_{0}, \lambda_{0}\right)$. The constant $D_{3}$ is given by $D_{3}=-6 C_{3} / C_{1}$.

The names of branching types follow Stuart [19].

To investigate the type of bifurcation at $\left(0, p_{0}\right)$ by using the above scheme, we have to compute the constants

$$
\begin{aligned}
& C_{1}=E_{x x \alpha}^{\prime \prime \prime}\left(0, p_{0}\right) e e \\
& C_{1}^{\prime}=E_{x x \beta}^{\prime \prime \prime}\left(0, p_{0}\right) e e \\
& C_{2}=E_{x x x}^{\prime \prime \prime}\left(0, p_{0}\right) e e e \\
& C_{3}=E_{x x x x}^{(4)}\left(0, p_{0}\right) e e e e+3 E_{x x x}^{\prime \prime \prime}\left(0, p_{0}\right) e e y_{0},
\end{aligned}
$$

where $y_{0}=\left(y_{01}, y_{02}\right)$ is a unique solution of the linear equation

$$
F_{x}^{\prime}\left(0, p_{0}\right) y_{0}+\left(y_{0}, e\right)_{H} e=-F_{x x}^{\prime \prime}\left(0, p_{0}\right) e e .
$$


The constants $C_{1}$ and $C_{1}^{\prime}$ have been calculated in the proofs of Theorems 5.2 and 5.3 respectively. Recall that

$$
C_{1}= \begin{cases}2 a_{0} & \text { if } \alpha_{0}^{2}>\beta_{0} \text { and } \Delta e_{1}-a_{0} e_{1}=0, \\ 2 b_{0} & \text { if } \alpha_{0}^{2}>\beta_{0} \text { and } \Delta e_{1}-b_{0} e_{1}=0, \\ -2 \alpha_{0} & \text { if } \alpha_{0}^{2}=\beta_{0},\end{cases}
$$

and $C_{1}^{\prime}=1$. Moreover, $C_{1}<0$. We sketch the method of finding $C_{2}$ and $C_{3}$. By the formula

$F_{x}^{\prime}\left(x, p_{0}\right) h=\left(\Delta^{2} z-[z, \sigma]-[w, \eta]+2 \alpha_{0} \Delta z+\beta_{0} z-3 \gamma w^{2} z,-\Delta^{2} \eta-[w, z]\right)$, where $x=(w, \sigma), h=(z, \eta)$, we obtain

$$
F_{x x}^{\prime \prime}\left(0, p_{0}\right) e e=\lim _{t \rightarrow 0} \frac{F_{x}^{\prime}\left(t e, p_{0}\right) e-F_{x}^{\prime}\left(0, p_{0}\right) e}{t}=\left(0,-\left[e_{1}, e_{1}\right]\right)
$$

and

$$
F_{x x x}^{\prime \prime \prime}\left(0, p_{0}\right) e e=\lim _{t \rightarrow 0} \frac{F_{x x}^{\prime \prime}\left(t e, p_{0}\right) e e-F_{x x}^{\prime \prime}\left(0, p_{0}\right) e e}{t}=\left(-6 \gamma e_{1}^{3}, 0\right) .
$$

Next, from the equality

$$
E_{x}^{\prime}\left(x, p_{0}\right) h=\left(F\left(x, p_{0}\right), h\right)_{H}
$$

we get

$$
\begin{aligned}
E_{x x}^{\prime \prime}\left(0, p_{0}\right) e e & =\left(F_{x}^{\prime}\left(0, p_{0}\right) e, e\right)_{H}, \\
E_{x x x}^{\prime \prime \prime}\left(0, p_{0}\right) e e e & =\left(F_{x x}^{\prime \prime}\left(0, p_{0}\right) e e, e\right)_{H}, \\
E_{x x x}^{\prime \prime \prime}\left(0, p_{0}\right) e e y_{0} & =\left(F_{x x}^{\prime \prime}\left(0, p_{0}\right) e e, y_{0}\right)_{H}, \\
E_{x x x x}^{(4)}\left(0, p_{0}\right) e e e e & =\left(F_{x x x}^{\prime \prime \prime}\left(0, p_{0}\right) e e e, e\right)_{H} .
\end{aligned}
$$

Substituting $F_{x}^{\prime}\left(0, p_{0}\right) e, F_{x}^{\prime}\left(0, p_{0}\right) y_{0},(26)$ and (27) into the last equalities and into (25) we get

$$
C_{2}=0 \quad \text { and } \quad C_{3}=-\frac{6}{\pi} \iint_{\Omega} \gamma e_{1}^{4} d u d v-\frac{1}{\pi} \iint_{\Omega}\left[e_{1}, e_{1}\right] y_{02} d u d v
$$

and

$$
\left\{\begin{array}{l}
\Delta^{2} y_{01}+2 \alpha_{0} \Delta y_{01}+\beta_{0} y_{01}+\left(\frac{1}{\pi} \iint_{\Omega} y_{01} e_{1} d u d v\right) e_{1}=0 \\
-\Delta^{2} y_{02}=\left[e_{1}, e_{1}\right] .
\end{array}\right.
$$

In consequence,

$$
C_{3}=-\frac{6}{\pi} \iint_{\Omega} \gamma e_{1}^{4} d u d v+\frac{1}{\pi} \iint_{\Omega}\left(\Delta^{2} y_{02}\right) y_{02} d u d v
$$

Finally, using Lemma 2.3 we obtain

$$
C_{3}=-\frac{6}{\pi} \iint_{\Omega} \gamma e_{1}^{4} d u d v+\frac{1}{\pi} \iint_{\Omega}\left(\Delta y_{02}\right)^{2} d u d v .
$$


Theorem 6.1 and the above computations lead us to the following conclusions.

Conclusion 6.2. Let $D=-6 C_{3} / C_{1}$, where $C_{1}, C_{3}$ are defined by (24). The point $\left(0, \alpha_{0}\right)$ is a bifurcation point of the equation (20) and the solution set of (20) in a small neighbourhood of $\left(0, \alpha_{0}\right)$ is the union of the trivial family

$$
\Gamma_{1}: \quad x_{1}(\alpha)=0, \quad \alpha \in\left(\mathbb{R}_{+}\right)_{\varepsilon}\left(\alpha_{0}\right),
$$

and a $C^{\infty}$ family $\Gamma_{2}$ which intersects $\Gamma_{1}$ at $\left(0, \alpha_{0}\right)$ only. Furthermore:

(i) If $C_{3}<0$ then $\Gamma_{2}$ can be parametrized as follows:

$\Gamma_{2}: \quad x_{2}^{ \pm}(\alpha)= \pm \sqrt{|D|}\left(\alpha_{0}-\alpha\right)^{1 / 2} e+o\left(\left|\alpha-\alpha_{0}\right|^{1 / 2}\right), \quad \alpha \in\left(\alpha_{0}-\varepsilon, \alpha_{0}\right]$,

(ii) If $C_{3}>0$ then $\Gamma_{2}$ can be parametrized as follows:

$$
\Gamma_{2}: \quad x_{2}^{ \pm}(\alpha)= \pm \sqrt{D}\left(\alpha-\alpha_{0}\right)^{1 / 2} e+o\left(\left|\alpha-\alpha_{0}\right|^{1 / 2}\right), \quad \alpha \in\left[\alpha_{0}, \alpha_{0}+\varepsilon\right) .
$$

Conclusion 6.3. Let $D^{\prime}=-6 C_{3}$, where $C_{3}$ is given by (24). The point $\left(0, \beta_{0}\right)$ is a bifurcation point of the equation (21) and the solution set of $(21)$ in a small neighbourhood of $\left(0, \beta_{0}\right)$ is the union of the trivial family

$$
\widehat{\Gamma}_{1}: \quad \widehat{x}_{1}(\beta)=0, \quad \beta \in\left(\mathbb{R}_{+}\right)_{\varepsilon}\left(\beta_{0}\right),
$$

and a $C^{\infty}$ family $\widehat{\Gamma}_{2}$ which intersects $\widehat{\Gamma}_{1}$ at $\left(0, \beta_{0}\right)$ only. Moreover:

(i) If $C_{3}<0$ then $\widehat{\Gamma}_{2}$ can be parametrized as follows:

$\widehat{\Gamma}_{2}: \quad \widehat{x}_{2}^{ \pm}(\beta)= \pm \sqrt{D^{\prime}}\left(\beta-\beta_{0}\right)^{1 / 2} e+o\left(\left|\beta-\beta_{0}\right|^{1 / 2}\right), \quad \beta \in\left[\beta_{0}, \beta_{0}+\varepsilon\right)$,

(ii) If $C_{3}>0$ then $\widehat{\Gamma}_{2}$ can be parametrized as follows:

$$
\widehat{\Gamma}_{2}: \quad \widehat{x}_{2}^{ \pm}(\beta)= \pm \sqrt{\left|D^{\prime}\right|}\left(\beta_{0}-\beta\right)^{1 / 2} e+o\left(\left|\beta-\beta_{0}\right|^{1 / 2}\right), \quad \beta \in\left(\beta_{0}-\varepsilon, \beta_{0}\right] .
$$

A task for future research is to check whether bifurcation occurs at points $(0, p) \in \Gamma$ such that $\operatorname{dim} N(p)>1$.

\section{References}

[1] S. S. Antman, Nonlinear Problems of Elasticity, Ser. Appl. Math. Sci. 107, Springer, 1995.

[2] A. Yu. Borisovich, Functional-topological properties of the Plateau operator and applications to the study of bifurcations in problems of geometry and hydrodynamics. Minimal surfaces, Adv. Soviet Math. 15 (1993), 287-330.

[3] -, Bifurcations of the capillary minimal surface in small gravitational fields, Mat. Sb. 188 (1997), 341-370.

[4] - Some scheme of applications of Crandall-Rabinowitz bifurcation theorem in variational problems of mechanics, preprint 135, Gdańsk Univ., 2000.

[5] A. Yu. Borisovich, Yu. Morozov and C. Szymczak, Bifurcations of the forms of equilibrium of nonlinear elastic beam lying on the elastic base, preprint 136, Gdańsk Univ., 2000. 
[6] C. S. Chien and M. S. Chen, Multiple bifurcation in the von Kármán equations, SIAM J. Sci. Comput. 6 (1997), 1737-1766.

[7] S. N. Chow and J. K. Hale, Methods of Bifurcation Theory, Springer, 1982.

[8] P. G. Ciarlet et P. Rabier, Les Équations de von Kármán, Lecture Notes in Math. 826, Springer, 1980.

[9] M. G. Crandall and P. H. Rabinowitz, Bifurcation from simple eigenvalues, J. Funct. Anal. 8 (1971), 321-340.

[10] E. J. Hölder and D. Schaffer, Boundary conditions and mode jumping in the von Kármán equations, SIAM J. Math. Anal. 3 (1984), 446-457.

[11] E. M. Kramer, The von Kármán equations, the stress function and elastic ridges in high dimensions, J. Math. Phys. 2 (1997), 831-846.

[12] J. E. Marsden, On the geometry of the Liapunov-Schmidt procedure, in: Lecture Notes in Math. 755, Springer, 1979, 77-82.

[13] Yu. Morozov, The study of the nonlinear model which describes the equilibrium forms, fundamental frequencies and modes of oscillations of a finite beam on an elastic foundation, Ph.D. Thesis, Appl. Math. Department, Voronezh University, 1998 (in Russian).

[14] L. Nirenberg, Topics in Nonlinear Functional Analysis, Courant Inst. of Math. Sci., 1974 .

[15] T. Poston and I. Stewart, Catastrophe Theory and its Applications, Pitman, 1978.

[16] B. Rao, Marguerre-von Kármán equations and membrane model, Nonlinear Anal. 8 (1995), 1131-1140.

[17] Yu. I. Sapronov, Branching of Solutions of Smooth Fredholm Equations, Lecture Notes in Math. 1108, Springer, 1982.

[18] —, Finite-dimensional reductions in smooth extremal problems, Uspekhi Mat. Nauk 51 (1996), no. 1, 101-132 (in Russian).

[19] C. A. Stuart, An introduction to bifurcation theory based on differential calculus, in: Nonlinear Analysis and Mechanics: Heriot-Watt Symposium, Vol. IV, Res. Notes in Math. 39, Pitman, 1979, 76-135.

[20] V. A. Trenogin and M. M. Vaŭnberg, Theory of Branching of Solutions of Nonlinear Equations, Nauka, 1969 (in Russian).

[21] I. I. Voronovich, Mathematical Problems in the Nonlinear Theory of Shells, Nauka, 1989 (in Russian).

Institute of Mathematics

University of Gdańsk

Wita Stwosza 57

80-952 Gdańsk, Poland

E-mail: jjancz@math.univ.gda.pl

Reçu par la Rédaction le 4.1.2001

Révisé le 6.6.2001 